DEVELOPMENT

\section{Slinging out old theories}

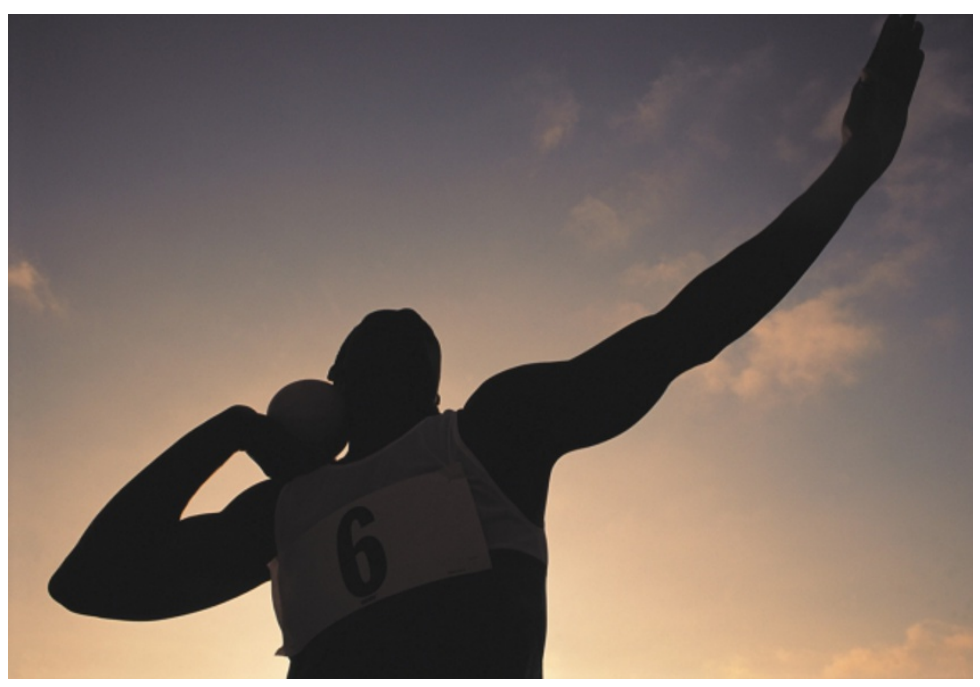

The glial sling - a transient bridgelike structure that spans the septum of the developing mammalian forebrain - is something of an enigma. Since it was discovered in the early 1980s, it has been assumed to consist of glioblasts, which were thought to provide a guidance substrate for the axons of the corpus callosum. However, because of a lack of suitable molecular markers, this idea was never corroborated. Now, as reported in Development, Shu and colleagues have revisited the glial sling in the mouse, using the array of markers that has since become available, and they present compelling evidence that it actually consists of migratory neurons.

Shu et al. found that the sling did not stain positive for commonly used glial markers such as GFAP, RC2 or GLAST. Markers for mature neurons, such as neurofilament, were also absent. However, the sling did express the early neuronal markers NeuN and TUJ1, indicating that the cells were most likely to be immature neurons. The sling cells also showed electrophysiological activity that was consistent with an early neuronal identity, including spikes that resembled $\mathrm{Na}^{+}$- and $\mathrm{Ca}^{2+}$-mediated action potentials. By filling the cells with the tracer biocytin, the authors showed that they had a neuronal morphology, with a long leading process and shorter processes that resembled dendrites.

Most of the neurons in the sling seem to originate from the cortical subventricular zone (SVZ), although Shu et al. also found evidence of cell proliferation within the sling itself. Interestingly, in spite of this proliferation, and the presumed continuous replenishment from the SVZ, the sling does not increase significantly in size during development. On the contrary, it begins to shrink after birth, and it is undetectable by postnatal day 10 .

\title{
Repulsive or attractive?
}

For neuronal networks to form correctly during development, nerve growth cones must respond precisely to extracellular guidance factors such as netrin-1. The repulsive and attractive guidance of axons by extracellular guidance factors is known to be modulated by intracellular second messengers, cyclic nucleotides and $\mathrm{Ca}^{2+}$. But how do these signalling molecules interact to determine the polarity of the guidance response? In Nature, Hong and colleagues now provide insights. A netrin-1 gradient induces the attraction of Xenopus spinal neuron growth cones through its receptor deleted-in-colorectal-cancer (DCC). However, when another netrin-1 receptor, UNC5, is present, netrin- 1 induces repulsion by acting through the DCC-UNC5 receptor complex. So, what are the intracellular signals that underlie these responses?

The authors first examined the effects of cyclic AMP and cyclic GMP signalling on DCC-mediated attraction in control neurons, and on DCC-UNC5-mediated repulsion in neurons overexpressing UNC5. Using a cGMP analogue and inhibitors of PKA (cAMP-dependent protein kinase) and PKG (cGMP-dependent protein kinase), they showed that cGMP signalling has an important regulatory role in netrin-1induced repulsion, whereas cAMP signalling modulates both DCC-mediated attraction and DCC-UNC5-mediated repulsion.

Next, Hong and co-workers studied the relative roles of CAMP and cGMP in netrin-1mediated growth-cone guidance. They varied the ratio of a cAMP and a cGMP analogue, and found that the growth-cone turning response correlated with this ratio; a high cAMP:cGMP ratio favoured attraction, whereas a low ratio favoured repulsion. But how does this cAMP:cGMP ratio modulate netrin-1 signals?

Netrin-1-induced attraction requires $\mathrm{Ca}^{2+}$ entry through channels such as L-type $\mathrm{Ca}^{2+}$ channels (LCCs), which are activated by cAMP and inactivated by cGMP signalling pathways. Hong and colleagues found that netrin-1 increased the amplitude of LCCmediated $\mathrm{Ca}^{2+}$ currents in control growth cones, whereas it decreased these currents in UNC5-overexpressing growth cones. Furthermore, they showed that a cGMP analogue and a PKA inhibitor decreased the $\mathrm{Ca}^{2+}$ currents in control neurons, and that a
PKG inhibitor rescued these currents in UNC5-overexpressing neurons. These results indicate that cyclic nucleotide signalling modulates LCC activity in nerve growth cones. So, in the model proposed by Hong and coworkers, netrin-1 activation of DCC leads to the activation of LCCs. DCC activation also leads to the activation of cAMP signalling, which, in turn, enhances LCC activity. This results in increased $\mathrm{Ca}^{2+}$ currents and growth-cone attraction. In the presence of UNC5, netrin-1 activation of DCC-UNC5 also activates CGMP signalling. This results in decreased $\mathrm{Ca}^{2+}$ currents and growth-cone repulsion. This work has therefore shown that interactions between cyclic nucleotide and $\mathrm{Ca}^{2+}$ signalling pathways are crucial for netrin-1-mediated axon guidance, and that the regulation of $\mathrm{Ca}^{2+}$ channels is an important early event in the transduction of netrin-1 signals.

Rachel Smallridge, Senior Editor, Nature Reviews Molecular Cell Biology

(10) References and links ORIGINAL RESEARCH PAPER Nishiyama, M. et al. Cyclic ORIGINAL RESEARCH PAPER Nishiyama, M. et al. Cyclic
AMP/GMP-dependent modulation of $\mathrm{Ca}^{2+}$ channels sets the polarity of nerve growth-cone turning. Nature 26 June 2003 (doi:10.1038/nature01751) 